DIVISION OF THE HUMANITIES AND SOCIAL SCIENCES

CALIFORNIA INSTITUTE OF TECHNOLOGY

PASADENA, CALIFORNIA 91125

MENTAL PROCESSES AND STRATEGIC EQUILIBRATION: AN FMRI STUDY OF SELLING STRATEGIES IN SECOND PRICE AUCTIONS

David M. Grether

California Institute of Technology

Charles R. Plott

California Institute of Technology

Daniel B. Rowe

Medical College of Wisconsin

Martin Sereno

University of California San Diego

John M. Allman

California Institute of Technology

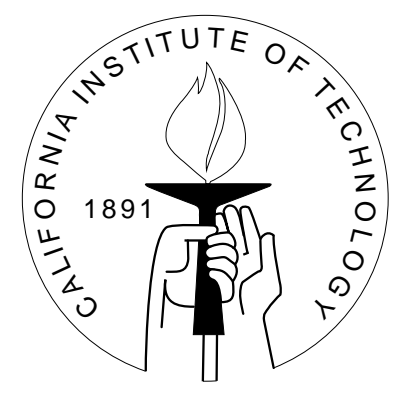

SOCIAL SCIENCE WORKING PAPER 1189R

February 2004

Revised June 2004 


\title{
Mental Processes and Strategic Equilibration: An fMRI Study of Selling Strategies in Second Price Auctions
}

\author{
David M. Grether \\ Charles R. Plott \\ Daniel B. Rowe \\ Martin Sereno \\ John M. Allman
}

\begin{abstract}
This study is the first to attempt to isolate a relationship between cognitive activity and equilibration to a Nash Equilibrium. Subjects, while undergoing fMRI scans of brain activity, participated in second price auctions against a single competitor following predetermined strategy that was unknown to the subject. For this auction there is a unique strategy that will maximize the subjects' earnings, which is also a Nash equilibrium of the associated game theoretic model of the auction. As is the case with all games, the bidding strategies of subjects participating in second price auctions most often do not reflect the equilibrium bidding strategy at first but with experience, typically exhibit a process of equilibration, or convergence toward the equilibrium. This research is focused on the process of convergence.

In the data reported here subjects participated in sixteen auctions, after which all subjects were told the strategy that will maximize their revenues, the theoretical equilibrium. Following that announcement, sixteen more auctions were performed. The question posed by the research concerns the mental activity that might accompany equilibration as it is observed in the bidding behavior. Does brain activation differ between equilibrated and non-equilibrated in the sense of a bidding strategy? If so, are their differences in the location of activation during and after equilibration? We found significant activation in the frontal pole especially in Brodmann's area 10, the anterior cingulate cortex, the amygdala and the basal forebrain. There was significantly more activation in the basal forebrain and the anterior cingulate cortex during the first sixteen auctions than in the second sixteen. The activity in the amygdala shifted from the right side to the left after the solution was given.
\end{abstract}

JEL classification numbers: C91, D83, C23

Key words: auctions, strategy, imaging 


\title{
Mental Processes and Strategic Equilibration:
}

\author{
An fMRI Study of Selling Strategies in Second Price Auctions ${ }^{4}$ \\ David M. Grether, Charles R.Plott, Daniel B. Rowe, \\ Martin Sereno, and John M. Allman
}

\section{INTRODUCTION}

The development of tools to measure reactions associated with mental activity opens the possibilities of fruitful interactions between economists and biologists, especially neuroscientists. This study is the first to join that interface with a focus on equilibration. Economic theory is typically a theory of equilibrium and equilibration for individuals, markets or, more generally, systems. While both market models and game theory models have both equilibrium and equilibration defined in terms of observable variables, the theory itself, when applied to the individual, is often developed in terms of the unobservable beliefs and objectives that the individual might hold. Thus, the theory is often developed from assumptions about unobservable states of mind along with the use of "as if" assumptions, to which theorists often resort when confronted by unobservables.

The application of the resulting "as if" methodology is widespread, well understood and remarkably successful in developing models for the behavior of both individuals and complex systems of individuals. Included are postulates of optimizing behavior and strategy, as well as postulates about the consequences of strategies that others might employ. Intentions, attributions of intentions and strategic thinking, none of which can be observed, play a role in the theory. At the base of the success of the theory, in spite of the lack of observability of key variables, rests a theory of equilibrium and equilibration. It is that particular feature of this complex that we focus on here. Can we observe mental activities that can be interpreted as equilibration that take place when observed choices would also be so interpreted?

Developments in the use of laboratory techniques in experimental economics together with the technological advancements of fMRI make possible collaboration between economics and neuroscience (Glimcher 2003, McCabe 2001) that might narrow the scope for speculation about what is taking place in the mind as economic decisions are made.

Distinguishing between equilibrium behavior and out of equilibrium behavior could have important implications for the study of economics. First, economic theory has little to say about behavior out of equilibrium. Thus, observing behavior of individuals who are not equilibrated does not provide a valid test of the theory. Knowing which data represent equilibrium behavior and which do not would be a significant breakthrough. The implications of commonly observed behavior that appears inconsistent with economic theory (Camerer, 1995), are quite different if the behavior is equilibrium behavior as opposed to confused responses of subjects searching for a solution (Plott, 1996).

\footnotetext{
${ }^{4}$ The research support of the Caltech Laboratory for Experimental Economics and the National Science Foundation are gratefully acknowledged.
} 
In this paper, we report the results of experiments conducted while subjects were inside a MRI scanner. Subjects participated in a series of auctions in which the optional response is to accurately reveal preferences, which seem obvious once understood.

Indeed, with training which sometimes includes explaining the optimal strategy, subjects typically adopt the strategy and in some cases do so only after a few trials. In the experiments conducted, optimal and non-optimal strategies are easily observed and then can be used to distinguish between equilibrium and disequilibrium behavior. Such decisions are made while the subject is undergoing fMRI so the differences in mental process during and after equilibration can be observed

\section{EXPERIMENTAL PROCEDURES}

\section{The Task}

The subject is given a coupon with a face value stated in the experimental currency called francs. Francs were converted to dollars at the end of the experiment at a fixed known exchange rate $(300 \mathrm{f}=\$ 1)$. The subject may keep the coupon and redeem it for cash at the end of the experiment or sell it and earn the sales price instead. The subject's task is to state a reservation price, i.e. the lowest price at which the subject is willing to sell the coupon. That price will be compared with a bid randomly generated independently of the subject's price. If the random bid is above the reservation price, the subject sells the coupon at the higher price. Otherwise the subject keeps the coupon. The experimental task is based upon the method developed by Becker, De Groot and Marshak (1964) for determining subjects' valuations of objects. We refer to this procedure as BDM. We used francs rather than direct cash values so that if we alter the design and change the number of tasks in a session the exchange rate can be adjusted to keep subjects earnings at a reasonable level. In practice, subjects earned about $\$ 70$ for the session, which ran about an hour and a half.

If one wants to maximize rewards, the optimal strategy is to be "truthful" in the sense of bidding exactly the amount of the coupon. Thus, if the subjects name an amount less than the value to them of the coupon, they risk selling it for less than the value. On the other hand, if they name an amount that is more than the coupon is worth to them, there is the possibility of rejecting bids that they would prefer to having the coupon. By naming any amount other than the true value of the coupon they cannot gain anything, but may lose something.

Regardless of the simplicity, major elements of this problem are present in almost every economic decision. First, is a notion of independence between subjects' bids and the bids to which theirs will be compared. If the bids are somehow related, then subjects would have an incentive to place bids with an objective of influencing the future bids used by the experimenter. Secondly, there is a notion of optimizing behavior from the point of view of the subject, as opposed to the set of all bidders. A subject who is concerned about being "fair" or concerned about appearance could behave much differently from theoretical predictions. Third, is the recognition that one strategy is dominant: it is optimal regardless of the outcome of the randomness. Recognition of dominance is an exercise in abstract logic that leads to the conclusion of the type of strategy that is optimal. Such interactions among motive, understanding, logic, beliefs and strategy have always been a challenge to economists, who traditionally have only observed behaviors as data. 
Subjects make sixteen bidding decisions and after each are informed of the amount of money they accumulate as a result. After the first sixteen auctions, subjects were told the correct strategy, that is, the strategy that will ensure them the largest amount of money. Instructions are attached in the Appendix. These were read prior to any decision or experience in the machine. Once in the machine, the screens they saw are typical of the example in Figure 1. The Instructions about the optional strategy, which was after the first sixteen auctions, are attached at the end of the general instructions. The objective of the research reported here is to study the subjects while mental activity is taking place both before and after the instruction about strategy is given to them. Presumably, if the decisions of the subject are not equilibrated before the instruction is given to them, they would be so afterward. The research questions we address are twofold. What locations in the brain show activation in the task? Are there systematic differences in brain activation before and after subjects have found the solution to the problem?

\section{Session Procedures}

Subjects were recruited primarily from the upper level, undergraduate student population of University of California at San Diego. However, the subjects did include graduate students, a postdoctoral fellow and a working adult. Each subject was first given a safety orientation and, if willing, signed the consent form. Next the subject read the Keypad Instructions for operating the specially designed non-metallic fiber optic BRU and then practiced with the BRU while seated in front of a laptop with the keypad to the right of the computer. There are no metal parts that could cause interference. The unit has four buttons that can be used to correspond to any four keys on the keyboard. In these experimental sessions, the buttons on the BRU corresponded to: Left, Right, Up and Down. Numbers were displayed on the screen as four digit numbers with all columns initialized at zero. The Right (Left) button caused the cursor to move one column to the right (left) with a wrap around to the far left (right) if the cursor was already in the rightmost (leftmost) column. The Up (Down) button increased (decreased) the digit in the active column by one. All buttons had analogous wrap around features. When the subject was comfortable with the BRU the subject was given the selling instructions. At this point, any questions about the procedures were answered.

The subject was then positioned in the scanner with the BRU taped to the right side and the right arm taped on the BRU to alleviate the need to hold the arm still. The projected output from the laptop was aligned on a screen so that the subjects can see the information on the experimental tasks projected into the scanner. Once the subject was properly placed in the scanner and able to read information displayed the subject is given a second practice session practice with the BRU under the new condition of lying down in the scanner. As the positioning in the scanner takes several minutes and could be distracting, the instructions for the bidding task are summarized on a screen projected into the scanner (See Appendix instructions). At this point, the experiment, which consists of four scanning session is conducted.

Imaging was performed on a 1.5T Siemens magnet at the Thornton Hospital of UCSD. The functional images consisted of 24 axial planes each 64 by 64 . Individual voxels were 45 cubic mm measuring 3 by 3 in plane and 5mm thick. A custom program was written that alternately displayed a Bidding Task and a blank screen. The program also reads the subject's input from the BRU and records each keystroke, the time elapsed, 
the bids and earnings. The program includes a display calibration screen to align the projector and a Raw Display Program, which reads and displays the Raw Data (Supporting material).

\section{THE STATISTICAL MODEL}

The data are panel data with the unit of observation being $x(i, t)$, the hemodynamic response of voxel $i$ at time $t$. The basic model is (Rowe 2001, 2003):

$$
x(i, t)=a(i)+b(i) * t+c(i, 1) * S(1, t)+\ldots+c(i, k) * S(k, t)+\varepsilon(i, t) .
$$

In the equation, the $S(k, t)$ are the values of $k$ possibly unobserved stimuli often called reference functions that the voxels are responding to. Each voxel has its own intercept and trend as well as coefficients for contributions from the $\mathrm{k}$ responses to the stimuli or reference functions. We take the disturbances, $\varepsilon(i, t)$ to be matrix normal with contemporaneous covariance matrix $\Psi$ and uncorrelated over time.

If the reference functions are unobserved, the model is similar to a factor analysis model. In factor analysis it is usually assumed that the factors (reference functions in this application) are orthogonal. We do not impose that condition here, so the model is perhaps better described as a latent variable model.

We proceed in a Bayesian fashion, and quantify available knowledge in the form of priors for the models parameters. The regression coefficients are taken to be jointly normal with covariance matrix $\Psi$, and mean matrix $\mathrm{C}_{0}$. The reference functions are taken to be matrix normal with prior mean $\mathrm{S}_{0}$, the columns of which are the assumed to be boxcar functions representing the experimental treatments, and covariance matrix $\mathrm{R}$. Usual practice is to estimate differences in response by introducing the boxcar functions (so-called due to their shape) taking the value 1 during a treatment and -1 or 0 when the treatment is not on, shifting them to allow for the hemo-dynamic lag and smoothing or tapering them to reduce discontinuities. The Bayesian approach allows the data to determine the shape of the reference functions. For a fuller discussion, see Rowe (2000, 2001). For an elementary but elegant discussion of Bayesian inference see Edwards, Lindman and Savage (1963). The variance covariance matrices, $\Psi$ and $R$, are taken to have inverted Wishart distributions with parameters $(v, Q)$ and $(\eta, V)$ respectively. The number of components was initially taken to be one. Later we considered the possibility of two reference functions and assumed that each of the two possible values of $\mathrm{k}$ (the number of functions) had prior probability of one half. We use an empirical Bayes approach and take the prior means for the coefficients of the reference functions to be the estimated coefficients of the voxels' activations on boxcar. We obtain the conditional densities of the parameters given the values of the other parameters and the hyper parameters. We estimate the mode of the posterior distribution by the method of iterated conditional modes (see Lindley and Smith (1972) and Rowe (2001) for details), and we obtain our estimates of the reference function (s) from the posterior mode. We proceed conditional upon the reference functions that we estimated from the mode of the posterior distribution. That is, we look at the relationship between the hemodynamic responses in voxels and the statistically determined Bayesian reference functions, using the Bayesian reference functions in place of the boxcar functions.

The procedures for calculating the statistical significance of the activation were based on the AFNI program and related software. For each scan, we computed the 
histogram of the $\mathrm{t}$ statistics for the full brain and picked the value that corresponded to a fixed percentile of the distribution (typically $95 \%$ ). We defined regions of interest (ROIs) prior to the analysis, and only used regions we could identify without reference to activation maps. For the frontal pole, for example, we took to all of the brain forward of minus 55 (Talairach) on the y-axis. We identified all clusters of voxels in ROIs such that at each voxel in the cluster the $t$ statistic was at least as large as the cut off level. Voxels were defined as being in the same cluster if their centers were no more than $5.7 \mathrm{~mm}$ apart. We calculated the bandwidths of the Gaussian filter corresponding to the spatial correlations in the data using the program 3dFWHM. We simulated the clusters in the ROIs using areas of roughly the same configurations as the ROIs. In the simulations samples of independent standard normal variables are generated and smoothed with the Gaussian filter estimated from the data. Each simulation was preformed 10,000 times and the number of clusters tabulated by size of cluster. Rather than perform separate simulations for each scan, we performed the simulations using the maximal bandwidths from the four scans for each subject. As larger bandwidths lead to more clusters this procedure is conservative.

The procedures for calculating the differences in activation were similar. The time series for scans one and two were added and the series for scans three and four subtracted from them. The statistical model was then fit using the average overall subjects and scans of the Bayesian components as the functions in equation (1). Once these calculations were performed, the methods were the same as those described.

\section{RESULTS}

The results are partitioned into two subsections. The first is found in the daily behavior as viewed from the point of view of economics. The second subsection is related to mental activity as rendered from the fMRI.

\section{Bidding Results}

The task is one that we believed subjects would spend some time trying to find the correct strategy and, then, would spend the rest of the trials using the optimal strategy. Previous experiments suggested the task we chose. The purpose was to observe activity during a non-equilibrated period and a subsequent equilibrated period. The first result of this section demonstrates that equilibration processes, as seen by economic theory, were observed on average. Table 1 shows the time structure of absolute difference between bid and the value of the ticket for each subject. Compared are the first sixteen auctions with the last sixteen. Equilibration occurs when the difference is zero. For no subject is the median lower in the first sixteen and for most it is higher. The data can be viewed a different way in Figure 2. Shown there are the eight bids for each subject during each of the four sessions plotted against the value of the ticket for which the subject was bidding. Equilibrium theory predicts that all bids should lie on the 45 degree line. Subjects were told the equilibrium strategy at the start of the third series of eight. The equilibration tendencies are obvious in the data.

RESULT 1: Overall, subjects exhibited convergence to the equilibrium strategy with more rapid convergence exhibited after the domination features of the equilibrium strategy were explained. 
SUPPORT. Each of the 17 subjects participated in 32 auctions, producing a total of 544 bids. Of these, 241 were exactly equal to the value of the coupon. There were 75 bids equal to coupon value in the first 16 trials and 166 during the second 16 trials. All coupon values and the predetermined bids were in multiples of ten (subjects were not told this). Thus, any bid within 10 of the coupon value earned the same amount as bidding the value exactly. More bids, mainly from the second 16 trials (203 compared to 108) satisfied this criterion. The magnitudes of the differences were not large in general. Averaging over all subjects, the average differences were 42.3 points for the first eight trials and 47.9 for the second eight trials. The differences for the second half of the session were much smaller: 0.5 points for the last eight trials and 9.1 for the third eight (ignoring one outlier at 9000).•

The figure illustrates the existence of variability across subjects. One concern was that the task would be so obvious to the subjects that they would see the solution immediately and indeed for some subjects that was the case as is summarized by the next result and support.

RESULT 2: Convergence properties differ across the behavior of individuals.

SUPPORT. Of the seventeen subjects who participated in scans, four nearly always used the dominant strategy from the very beginning (subjects 2, 4, 6 and 11). An additional three subjects (subjects 3,9 and 14) converged to the dominant strategy without being told for a total of seven subjects that consistently used the dominant strategy without being given the solution. In addition, seven subjects followed the correct strategy after being given the solution. Even after being given the solution, subjects frequently experimented a bit before adopting the dominant strategy. All but three subjects adopted the dominant strategy by the end of the experiment (two consistently priced over the value and one priced below). Initial behavior, however, was heterogeneous. Six subjects began by pricing over the value, three consistently set prices below the value, four gave prices both over and under the value and four used the dominant strategy from the beginning.•

The source of the observed variablity differs from individual to individual and during the first phases appears to be related to strategic behavior based on misunderstandings or confusion about the full implication of various strategies. The misguided strategy seems to come in two forms. Some individuals place a higher bid believing they will receive a higher price not realizing that in a second price auction they are exposed to an opportunity for loss. Other subjects offer at low prices explaining the strategy by saying that they wanted to make the sale. Both properties can be seen in the first panels of Figure 2. Notice two separate groupings of data, one with a tendency to be above the $45^{\circ}$ line of the optimal strategy and the other much below the line. On average, there was a greater tendency to over bid with 205 prices set above coupon value and 95 
set below. ${ }^{5}$ Notice that in the lower panels the two groups have merged into one as subjects tended to adopt the optional strategy.

Those subjects who did not adopt the dominant strategy of giving the coupon value as their reservation price seemed to adopt some simple alternative e.g. rounding up the coupon value to the next multiple of 100, or alternating above and below. One subject alternated by being over the coupon value by 30 points and by under 70 points.

A scanner is hardly an ideal decision making environment. The subjects are motionless on their backs and in a tube looking at a screen through mirrored glasses. The button response unit (BRU) is taped to them, and the scanner, when active, makes a lot of noise. Given this, the overall performance is reasonable. Pilot experiments with subjects who were not in scanners exhibited similar convergence.

\section{Results from fMRI: Mental Activity Measurements}

The brain areas activated in these experiments include the frontal polar cortex, which is known from previous studies to be involved in calculation, and a group of brain structures that receive input from the dopaminergic neurons in the midbrain that signal expectation of reward. These structures include the basal forebrain, the amgdala and the anterior cingulate cortex.

Two additional results emerge from the brain scans. The first of these (Result 3) identify the location in which significant activities were observed. The second (Result 4) summarizes the changes in mental activity across the treatments.

Each subject participated in four sets of bids each eight minutes and thirty-two seconds long. The sequence was twenty-two seconds during which the value of the coupon was displayed (see supporting materials for the display) and the subject entered their reservation price, ten seconds for feedback, followed by thirty-two seconds of blank screen. For technical reasons we were unable to analyze the MRI data from the first 6 subjects; the analysis is based on subject 7 through 17.

RESULT 3. Significant activation occurs in four areas: the frontal pole (especially Brodmann's Area 10), the amygdala, the anterior cingulate cortex and the basal forebrain.

SUPPORT. The activations are summarized in Table 2. The table gives the estimated significance levels for the largest cluster of voxels in the indicated regions all of which exceeded a predetermined cutoff level. Frequently, there were multiple clusters found which were significant at conventional levels of significance. The significance levels shown are, therefore, conservative. As stated above, the anterior cingulate activity was greater in the first sixteen auctions, which is shown in Figure 3, a sagittal view (at $x=0)$ depicting the difference between the intensity over the first 16 rounds minus rounds 1732. The right amygdala was more active in the first 16 rounds than the left (see Figure 4).•

\footnotetext{
${ }^{5}$ Explanation of the tendency to over bid involves some controversy. Kahneman, Knitsch and Thaler (1991) interpret this as an inducement effort", on the other hand Plott and Zeiler (2003) suggest that it results from subject's misunderstandings of the task.
} 
RESULT 4. Activation levels changed over the course of the treatments. The anterior cingulate activity was greater during the first (during theoretical equilibration) trials as was the right amygdala. During the second sixteen trials (after theoretical equilibration), amygdalar activity shifted to the left side and the anterior cingulate activity reduced.

SUPPORT. The right amygdala was more active in the first 16 rounds than the left (see Figure 2). In the final rounds, the amygdalar activity shifted to the left side. Table 3 gives the significance levels for the differences in the responses between the first two scans (before subjects were given the solution to the problems) and the last two scans (after the solution was given). Table 4 contains the same information for the scans immediately before and after subjects were given the solution (scans 2 and 3). In the tables we report significance levels for the largest clusters of each sign (a minus sign indicates the cluster is significant with a negative sign indicating greater activation in the later scans) and the centers of mass of the clusters (weighted by intensity).•

\section{DISCUSSION}

The results reported here suggest the existence of a correspondence between the phenomenon of equilibration in decision rules as it is interpreted by economic theory and the physiological traces of mental processing. Such a correspondence holds a potential for joining two sciences in useful ways. On one hand, as already noted, a concept of equilibrium is central to much of economic theory. The theory interprets behavior "as if" certain mental processes were taking place and introspection about those processes often serves to motivate new theory. In the absences of direct observations of mental activity, competing interpretations of observed behavior are difficult to resolve. For example, an individual who is confused and exhibits unchanging behavior while resolving a decision problem could be interpreted as having attained equilibrium. Furthermore, measurements of behavior tend to be limited to those that are suggested by the theory, which is itself conditioned on an assumption that mental processes cannot be observed. Thus, for economics the discovery suggests the possibility of enriching the set of observable variables that might be interpreted by theory. Similarly, on the other hand, the discovery connects neuroscience with a new set of behaviors and suggests new interpretations of the areas of the brain in which mental activities are taking place.

The task was chosen so that we expected to image brain activity during a nonequilibrated or searching stage and after equilibration. To this end, the task was not perfect. If a subject happens to adopt the equilibrium strategy, by accident or without realizing that it is indeed a correct strategy, there was no way no way to tell. In our experiment, for example, it is possible that subjects could respond with the face value of the coupon because it is a salient number and not because they had determined that this was in fact the best possible response. Though a few subjects adopted the optimal strategy without being given the solution, all subjects were given the optimal strategy after the first two scans. We do not know if those subjects using the optimal strategy before getting the solution actually understood the task rather than responding with the coupon value as a focal point. 
With the qualifications as above, we conservatively identify observations from the second two scans as being equilibrated and those from the first two as being nonequilibrated. In this regard, we see more activation during the first two scans in the anterior cingulate and the right amygdala than during the later scans. Thus, we interpret the phenomena of disequilibration with the anterior cingulate, which dampens with the process of equilibration. The findings and interpretation are consistent with other findings reported in the literature.

The strongest and most consistent activation was located near the frontal pole, primarily in Brodmann's area 10 (Table 5). This area has been previously been found to be active in other tasks involving monetary reward and risk assessment (Rogers et al., 1999; Ernst et al., 2001). Area 10 is also activated in mental calculation (Rickard, 2000) which the subjects would have been performing in this task. Area 10 is activated when an expected financial reward is received (Knutson, et al. 2003). Area 10 is both absolutely and relatively much larger in humans than in other primates which suggests that some of the circuitry related to economic decision-making is a phylogenetic specialization in the human brain (Allman et al., 2002; Semendeferi et al., 2001).

The anterior cingulate cortex, basal forebrain, and amygdala all receive strong dopaminergic input from the midbrain, which signals the expectation of reward (Schultz, 2002). The anterior cingulate was active during the first 16 auctions before the subjects were told the optimal bidding strategy and less active during the second 16 auctions. The anterior cingulate cortex is active in difficult tasks involving considerable uncertainty (Critchley, et al. 2001). The anterior cingulate cortex is also active in tasks the require novel as opposed to routine solutions (Raichle et al, 1994). The anterior cingulate cortex is the source of an EEG signal associated with financial loss in gambling (Gehring and Willoughby, 2002), which would presumably not occur with the application of the optimal bidding strategy. More broadly speaking, the anterior cingulate cortex is involved in maintaining physiological homeostasis through the regulation of a wide variety of mechanisms necessary for life including hunger and thirst. (Liotti, et al. 2001). This broader view of a homeostatic role for anterior cingulate is consistent with the lack of activation in these structures during economic decision making during equilibrium conditions.

The basal forebrain contains many reward-related neurons (Schultz, 2000). Activity in the ventral striatal component of the basal forebrain is specifically related to cumulative financial reward during a series of gambles (Elliott et al., 2003). The amygdala is strongly associated with fear (Ledoux, 1995) and it is possible that the initial activation on the right side during the disequilibrium phase may have arisen from the subjects' fear of financial loss. However, the activation of the left amygdala during the second sixteen auctions after the subjects had been informed of the optimal strategy may be associated with their increased certitude of receiving financial reward. There is increasing evidence that amygdala is involved in positive reward assessment as well as its more familiar role in fear (Baxter and Murray, 2002; Schultz, 2002). Recently, Fried et al., (2001) have shown through micro dialysis that the dopaminergic input to the amygdala in human subjects increases during cognitive tasks not involving fear.

The fact that the location of neural activity changes as equilibration takes place means that in imaging studies, equilibration and time must be considered in addition to features of the task. Clearly there is much to be done. In pilot studies, we included control tasks, simple calculations requiring response on the BRU, and found that subjects 
were distracted by the second task, forgetting their experience in the earlier auctions. Thus, we did not include control tasks because of the expected interference in subjects' finding the optimal strategy in the selling task. A natural extension would be to bring subjects back after a few days to redo the task. Assuming that they then understand the task, we could use these extra trials to compare their now (presumed to be) equilibrated behavior with the scans from the earlier sessions. At this time it should be able to introduce other control tasks.

The task studied involves numerical responses. While this is true of many economic decisions, it is by no means true of all. Choices from non-numerically valued alternatives are basic to economics, and could also be studied. The task studied has a single individual acting alone with alternative bids predetermined by a computer. Bargaining, or any type of other regarding behavior is not included in the situation studied. A natural extension would be to have subjects buying and selling in markets with other participants. 


\section{REFERENCES}

Allman, J.M., Watson, K, and Hakeem, A. (2002) "Two phylogenetic specializations in the human brain", Neuroscientist 8:335-347.

Baxter, Mark G., and Murray, E.A. (2002) "The amygdala and reward", Nature Reviews, Neuroscience 3:563-573.

Critchley, HD, Mathias CJ, Dolan RJ. (2001) "Neural activity in the human brain relating to uncertainty and arousal during anticipation", Neuron. 2001 Feb;29(2):537-45.

Dishbrow, E.A. et al. (2000) "Functional MRI at 1.5 tesla: A comparison of the blood oxygenation level-dependent signal and electrophysiology: Proceedings of the National Academy of Sciences", 97:9718-23.

Edwards, W., Lindman H. and Savage L.J. "Bayesian statistical inference for psychological research", Psychological Review 70:193-242.

Elliott, R., Friston, K., and Dolan, R., (2000) "Dissociable neural responses in human reward systems", Journal of Neuroscience 20:6159-6165.

Elliott, R, Newman, J. Longe, O. and J. Deakin (2003) "Instrumental responding for rewards is associated with enhanced neuronal response in subcortical reward systems", Neuroimage. 2004 Mar;21(3):984-90.

Ellsberg. Daniel (1961) "Risk, ambiguity, and the Savage axions”, Quarterly Journal of Economics 75:643-669.

Ernst, $\mathrm{M}$ et al. (2001) "Decision-making in a risk-taking task: a PET study", Neuropsychopharmacology 26:682-691.

Fried, Itzhak et al. (2001) "Increased dopamine release in the human amygdala during performance of cognitive tasks", Nature Neuroscience 201-6.

Gehring, W.J. and Willoughby A.R. (2002) "The Medial Frontal Cortex and the Rapid Processing of Monetary Gains and Losses", Science 295:2279-2282.

Glimcher, Paul W. (2003) "Decisions, Uncertainty and the Brain: The Science of Neuroeconomics", MIT Press.

Kahneman, D., Knetsch, J.L.,Thaler, R.H., (1990) "Experimental Tests of the Endowment Effect and the Coarse Theorem", The Journal of Political Economy, 98:1325-48.

Kahneman, Daniel and Amos Tversky (1979) "Prospect Theory: An analysis of Decision under Risk", Econometrica 47:263-291.

Knutson B., Fong GW, Bennett S.M., Adams C.M., Hommer D., (2003) “A region of mesial prefrontal cortex tracks monetarily rewarding outcomes: characterization with rapid event-related fMRI", Neuroimage. 2003 Feb;18(2):263-72.

LeDoux, Joseph (1996) The Emotional Brain Simon \& Schuster, New York.

Lindley, D.V. and A.F.M. Smith (1972) "Bayes estimates for the linear model”, Journal of the Royal Statistical Society 34:1-19.

Liotti, M., Brannan, S., Egan, G., Shade R., Madden L., Abplanalp, B., Robillard, R., Lancaster, J., Zamarripa, F., Fox, O., and Denton, D. (2001) "Brain responses associated 
with consciousness of breathlessness (air hunger)", Proceeding of the National Academy of Sciences 98:2035-40.

McCabe, K., Houser, D., Ryan, L., Smith, V., Tromard, T. (2001) “A functional Imaging Study of Cooperation in Two-Person Reciprocal Exchange", Proceedings of the National Academy of Sciences 98 (20):11832-11835.

Myagkov, Mikail and Charles R. Plott (1997) "Exchange Economies and Loss Exposure: Experiments Exploring Prospect Theory and Competitive Equilibrium in Market Environments", American Economic Review 87:801-28.

Platt, M.L. and P.W. Glimcher (1999) "Neural correlates of decision variables in parietal cortex", Nature 400:233-238

Plott, C.R. (1996), "Rational Individual Behavior in Markets and Social Choice Processes: The Discovered Preference Hypothesis", The Rational Foundations of Economic Behavior, K.J. Arrow, E. Colombatto, M. Perlman and C. Schmidt (Editors).

Plott, C.R. and K. Zeiler (2003), "The Willingness to Pay/Willingness to Accept Gap, the 'Endowment Effect,' Subject Misconceptions and Experimental Procedures for Eliciting Valuations", American Economic Review (submitted).

Raichle, M., J.A. Feiz, T.O. Videen, A.M. MacLeod, J.V. Pardo, P.T. Fox \& S.E. Petersen (1994) "Practice-Related Changes in Human Brain Functional Anatomy During Nonmotor Learning", Cerebral Cortex 4:8-26.

Rees, G., Friston, K., and Koch, C. (2000) "A direct quantitative relationship between the functional properties of human and macaque", Nature Neuroscience 3:716-23.

Rickard, T., Romero S., Basso G., Wharton C., Flitman S., Grafman J. (2000) "The calculating brain: an fMRI study", Neuropsychologia 38:325-35

Rowe, D.B. (2001) "Bayesian Source Separation for Reference Function Determination in fMRI", Magnetic Resonance in Medicine 46:374-378.

Rowe, D.B (2003) Multivariate Bayesian Statistics: Models for Source Separation and Signal Unmixing, Chapman and Hall/CRC Press.

Russo, Eugene (2000) "Debating the Meaning of fMRI", The Scientist 14:18-20.

Schultz. et al. (2000) "Reward processing in primate orbital frontal cortex and basal ganglia", Cerebral Cortex 10:272-84.

Schultz, W. (2002) “Getting Formal with Dopamine and Reward”, Neuron 36: 241-263.

Semendeferi, K., Armstrong, E., Schleicher, A., Zilles, K., and Van Hoesen, G. (2001)

"Prefrontal cortex in humans and apes: a comparative study of area 10", American Journal of Physical Anthropology 114:224-241.

Smith, Kip et al. (2002) "Neuronal Substrates for Choice under Ambiguity, Risk, Certainty, Gains and Losses", Management Science 48:711-718.

Thut, G. et al. (1997) "Activation of the human brain by monetary reward", Neuroreport $8: 1225-1228$. 
Figure 1

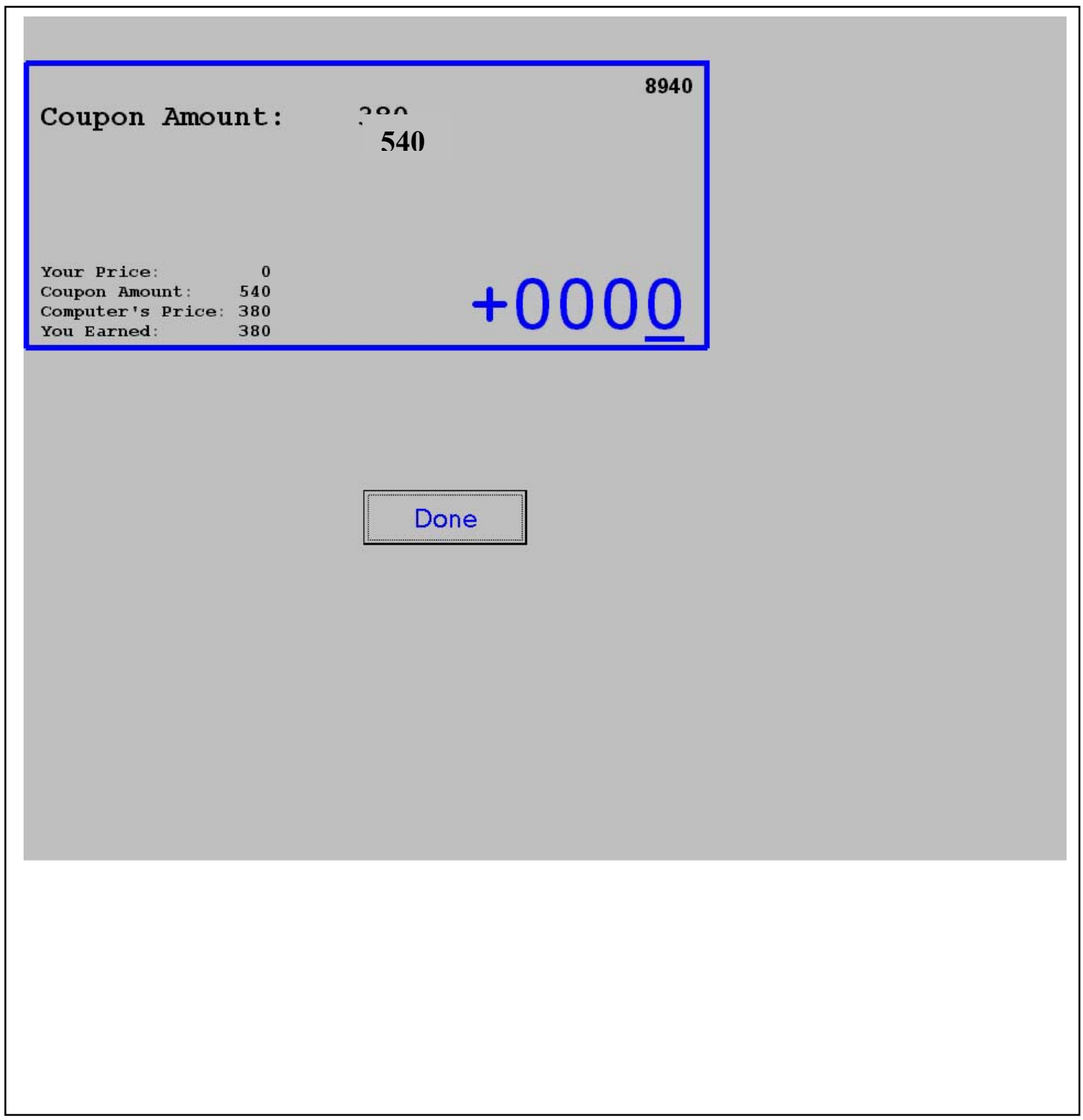

Sample Display 
Table: 1 Absolute Value of the Bid minus the Value of the Ticket

\begin{tabular}{|l|c|c|r|r|r|r|r|c|}
\cline { 2 - 9 } \multicolumn{1}{c|}{} & \multicolumn{4}{|c|}{ Auctions 1 to 16 } & \multicolumn{4}{c|}{ Auctions 17 to 32 } \\
\hline Subject & Mean & $\begin{array}{l}\text { Std. } \\
\text { Dev. }\end{array}$ & Median & $\begin{array}{l}\text { Number } \\
\leq 10\end{array}$ & Mean & $\begin{array}{l}\text { Std. } \\
\text { Dev. }\end{array}$ & Median & $\begin{array}{l}\text { Number } \\
\leq 10\end{array}$ \\
\hline 1 & 419.1 & 111.5 & 399 & 0 & 388 & 94.2 & 0 & 13 \\
\hline 2 & .1 & .3 & 0 & 16 & .2 & .5 & 0 & 16 \\
\hline 3 & 83.9 & 92.0 & 65 & 5 & 5.9 & 4.4 & 5 & 14 \\
\hline 4 & 23.3 & 84.5 & 1 & 15 & 1 & 0 & 1 & 16 \\
\hline 5 & 73.8 & 76.3 & 60 & 1 & 59.4 & 24.9 & 60 & 0 \\
\hline 6 & 0.0 & 0.0 & 0 & 16 & 0 & 0 & 0 & 16 \\
\hline 7 & 379.2 & 145.5 & 400 & 1 & 0 & 0 & 0 & 16 \\
\hline 8 & 187.3 & 97.9 & 210 & 0 & 15.7 & 28.0 & 5 & 11 \\
\hline 9 & 14.1 & 30.1 & 0 & 12 & 0 & 0 & 0 & 16 \\
\hline 10 & 78.8 & 35.8 & 80 & 1 & 580 & 2245.6 & 0 & 12 \\
\hline 11 & 6.3 & 3.6 & 0 & 16 & 0 & 0 & 0 & 16 \\
\hline 12 & 31.9 & 29.9 & 25 & 5 & 25.0 & 30.6 & 10 & 8 \\
\hline 13 & 263.0 & 161.7 & 278 & 1 & 129.7 & 125.9 & 95 & 2 \\
\hline 14 & 29.4 & 55.4 & 7.5 & 9 & 0 & 0 & 0 & 16 \\
\hline 15 & 51.6 & 22.5 & 50 & 0 & 50 & 26.7 & 50 & 0 \\
\hline 16 & 91.9 & 107.0 & 61.5 & 3 & 0 & 0 & 0 & 16 \\
\hline 17 & 167.1 & 224.4 & 17 & 7 & 53.8 & 215.0 & 0 & 15 \\
\hline
\end{tabular}

\footnotetext{
${ }^{6}$ Deleting one observation at 9000 reduces the mean and standard deviation to 18.7 and 38.9 , respectively.
} 
Table 2

Significance Levels of Responses for the Four Scans

\begin{tabular}{|c|c|c|c|c|c|}
\hline $\begin{array}{l}\text { Region } \\
\text { Subject }\end{array}$ & $\begin{array}{l}\text { Frontal } \\
\text { Pole }\end{array}$ & $\begin{array}{l}\text { Anterior } \\
\text { Cingulate } \\
\text { Cortex }\end{array}$ & $\begin{array}{l}\text { Basal } \\
\text { Forebrain }\end{array}$ & $\begin{array}{l}\text { Left } \\
\text { Amygdala }\end{array}$ & $\begin{array}{l}\text { Right } \\
\text { Amygdala }\end{array}$ \\
\hline$\overline{77}$ & $\begin{array}{l}\text { a a c a } \\
\text { a c d c }\end{array}$ & & $\begin{array}{l}a \mathrm{a} a \mathrm{a} \\
\mathrm{da}-\mathrm{b}\end{array}$ & $---a$ & $\begin{array}{l}\text { c d } \\
---d\end{array}$ \\
\hline 8 & $\begin{array}{l}a b a c \\
a a b b\end{array}$ & $\begin{array}{l}--d \\
b\end{array}$ & $\begin{array}{l}\mathrm{a} a \mathrm{a} a \\
\mathrm{~b} b \mathrm{a}\end{array}$ & $\begin{array}{l}\mathrm{b} b \mathrm{~b} c \\
\mathrm{c}-\mathrm{b}\end{array}$ & $-a$ \\
\hline 9 & $\begin{array}{l}c d b d \\
-a a a\end{array}$ & $\begin{array}{l}-\mathrm{c} \\
-\mathrm{a} \mathrm{a}\end{array}$ & $\begin{array}{l}\text { a a a a } \\
\text { a a - a }\end{array}$ & $\begin{array}{l}-c \\
-a c\end{array}$ & $\begin{array}{l}b-a c \\
c-c a\end{array}$ \\
\hline 10 & $\begin{array}{l}\text { a a a a } \\
\text { a c a a }\end{array}$ & $\begin{array}{l}\mathrm{c} b \mathrm{ad} \\
-\mathrm{c}-\mathrm{b}\end{array}$ & $\begin{array}{l}\text { c a a a } \\
- \text { a a a }\end{array}$ & $\begin{array}{l}--d b \\
-a b a\end{array}$ & $\begin{array}{l}a b \\
-a\end{array}$ \\
\hline 11 & $\begin{array}{l}\text { c a a a } \\
\text { a a a a }\end{array}$ & & $\begin{array}{l}\mathrm{b} a \mathrm{dc} \\
\mathrm{d}-\mathrm{d} b\end{array}$ & $\begin{array}{l}\text { a a b c } \\
\text { a c c a }\end{array}$ & \\
\hline 12 & $\begin{array}{l}\text { a a a } \\
\text { a a a a }\end{array}$ & $\begin{array}{l}a--b \\
a-b\end{array}$ & $\begin{array}{l}a--c \\
a b a d\end{array}$ & $\begin{array}{l}b-b \\
-c\end{array}$ & $\begin{array}{l}b-c \\
c-c\end{array}$ \\
\hline 13 & $\begin{array}{l}\text { a a a a } \\
\text { a c a a }\end{array}$ & $\begin{array}{l}-a d \\
--a\end{array}$ & $\begin{array}{l}-b \\
a d c\end{array}$ & c & $-c-c$ \\
\hline 14 & a a a a & $\begin{array}{l}-c-a \\
-h_{a}\end{array}$ & $--a \mathrm{a}$ & & $\ldots h$ \\
\hline 15 & ca a & $\begin{array}{r}\text { a c } \\
-h\end{array}$ & a ca c & $\begin{array}{l}-\mathrm{cc} \\
\mathrm{c}-\mathrm{ac}\end{array}$ & \\
\hline 16 & $\begin{array}{l}\text { a a a a } \\
\text { a a a a }\end{array}$ & $--\mathrm{a}$ & $\begin{array}{l}-\mathrm{a}-\mathrm{b} \\
\mathrm{c} \mathrm{ad}\end{array}$ & $c-a$ & $--a$ \\
\hline 17 & $\begin{array}{l}\text { a a a } \\
\text { b a a a }\end{array}$ & $\begin{array}{l}\text { a } \\
- \text { a a a }\end{array}$ & $\begin{array}{l}a b-c \\
a d a a\end{array}$ & & $\begin{array}{l}d-c \\
--d\end{array}$ \\
\hline
\end{tabular}

The letters in each cell give the significance levels for the four scans in order.

The first row gives significance levels for the first component, and the second row gives significance levels for the second component.

Legend: a $\mathrm{p}<.0005$; $\mathrm{b} \mathrm{p}<.005$; $\mathrm{c} \mathrm{p}<.05$; $\mathrm{d} \mathrm{p}<0.1$; blank or $-\mathrm{p}>.10$ 
Table 3

Response Scans $1 \& 2$ minus $3 \& 4$

Differences computed for each voxel

First Component

$\begin{array}{lcccc}\text { Subject } & \text { Frontal pole } & \text { Anterior Cingulate } & \text { Basal Forebrain } & \text { Amygdala } \\ 7 & \text { Cortex } & \text { b- } & \text { c } \\ 8 & \text { a, a- } & & \text { a } & \text { a } \\ 9 & \text { a,a- } & \text { d } & \text { a, }- & \\ 10 & \text { a,a- } & & \text { a- } & \text { a } \\ 11 & \text { a- } & \text { a } & \text { c } & \\ 12 & \text { a,a- } & \text { b } & \text { a } & \\ 13 & \text { a,a- } & & & \\ 14 & & \text { a } & & \\ 15 & \text { a,a- } & \text { Second Component } & \\ 16 & \text { a,a- } & & \end{array}$
Subject Frontal pole Anterior Cingulate Basal Forebrain Amygdala Cortex

$\begin{array}{ll}7 & \text { a,a- } \\ 8 & \text { c } \\ 9 & \end{array}$
10 a,a- a-
11 a,a- a
12 a,a- a,a- b,a- a-

13 a,a- a
14 a,a- a-
15 a-
16
17 c, a-

Legend: a $\quad \mathrm{p}<.0005 ; \mathrm{b} \quad \mathrm{p}<.005 ; \mathrm{c} \quad \mathrm{p}<.05 ; \mathrm{d} \quad \mathrm{p}<.10$ 
Table 4

Response Scan 2 minus Scan 3

Differences computed for each voxel

First Component

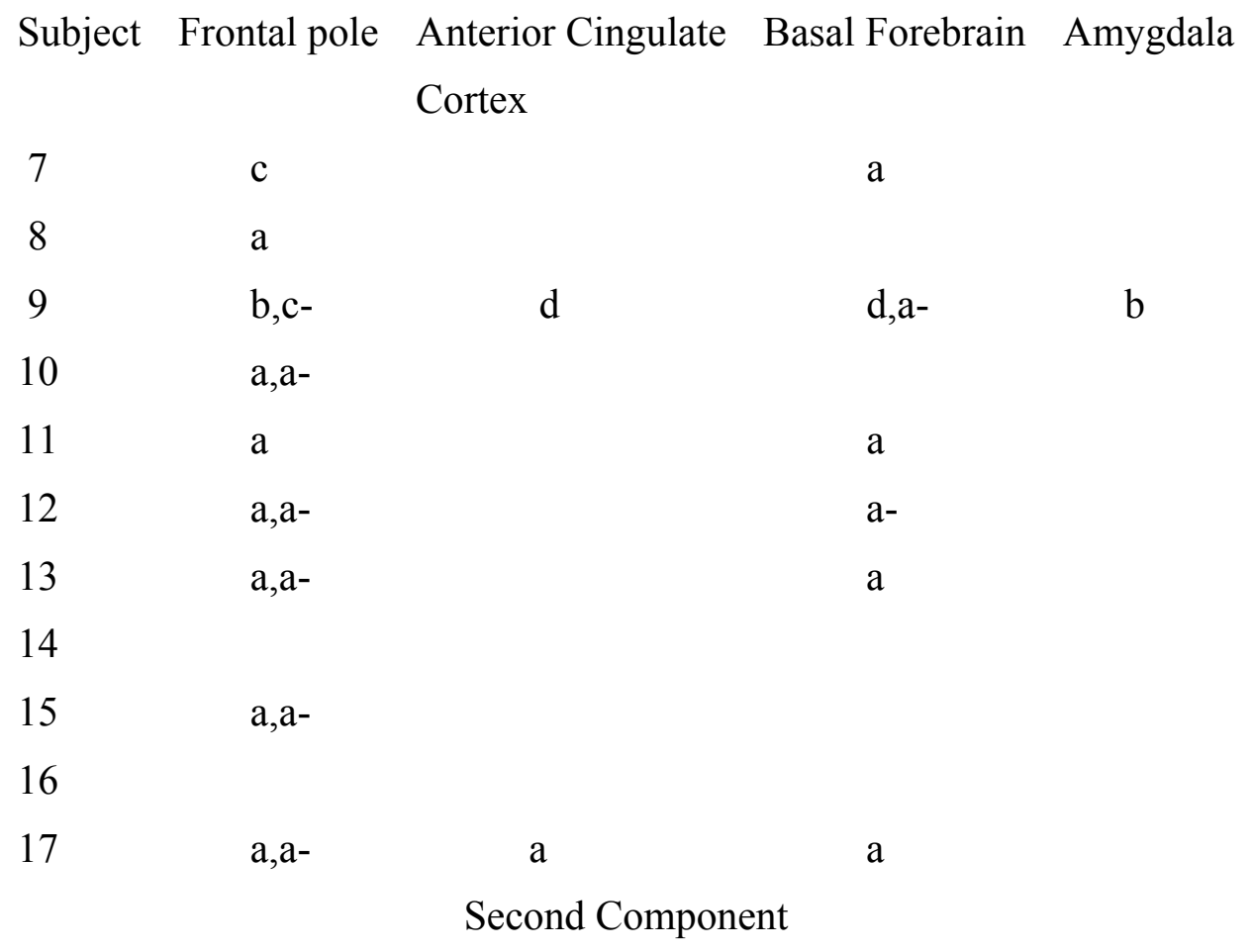

Subject Frontal pole Anterior Cingulate Basal Forebrain Amygdala Cortex

$\begin{array}{llll}7 & \text { a, } & \text { a } & \text { a } \\ 8 & \text { a } & \text { a } & \text { b,b- } \\ 9 & \text { a,a- } & \text { a,a- } & \\ 10 & \text { a,a- } & \text { a- } & \\ 11 & \text { a- } & \text { a- } & \text { d } \\ 12 & \text { c- } & & \text { a } \\ 13 & \text { a,a- } & \text { a,a- } & \\ 14 & \text { a,a- } & & \\ 15 & \text { a,a- } & & \end{array}$

Legend: a $\quad \mathrm{p}<.0005 ; \mathrm{b} \quad \mathrm{p}<.005 ; \mathrm{c} \quad \mathrm{p}<.05 ; \mathrm{d} \quad \mathrm{p}<.10$ 
Table 5

Area 10 Significant Activation

Component 1

Scan $1 \quad 2 \quad 3 \quad 4$

Subject 7

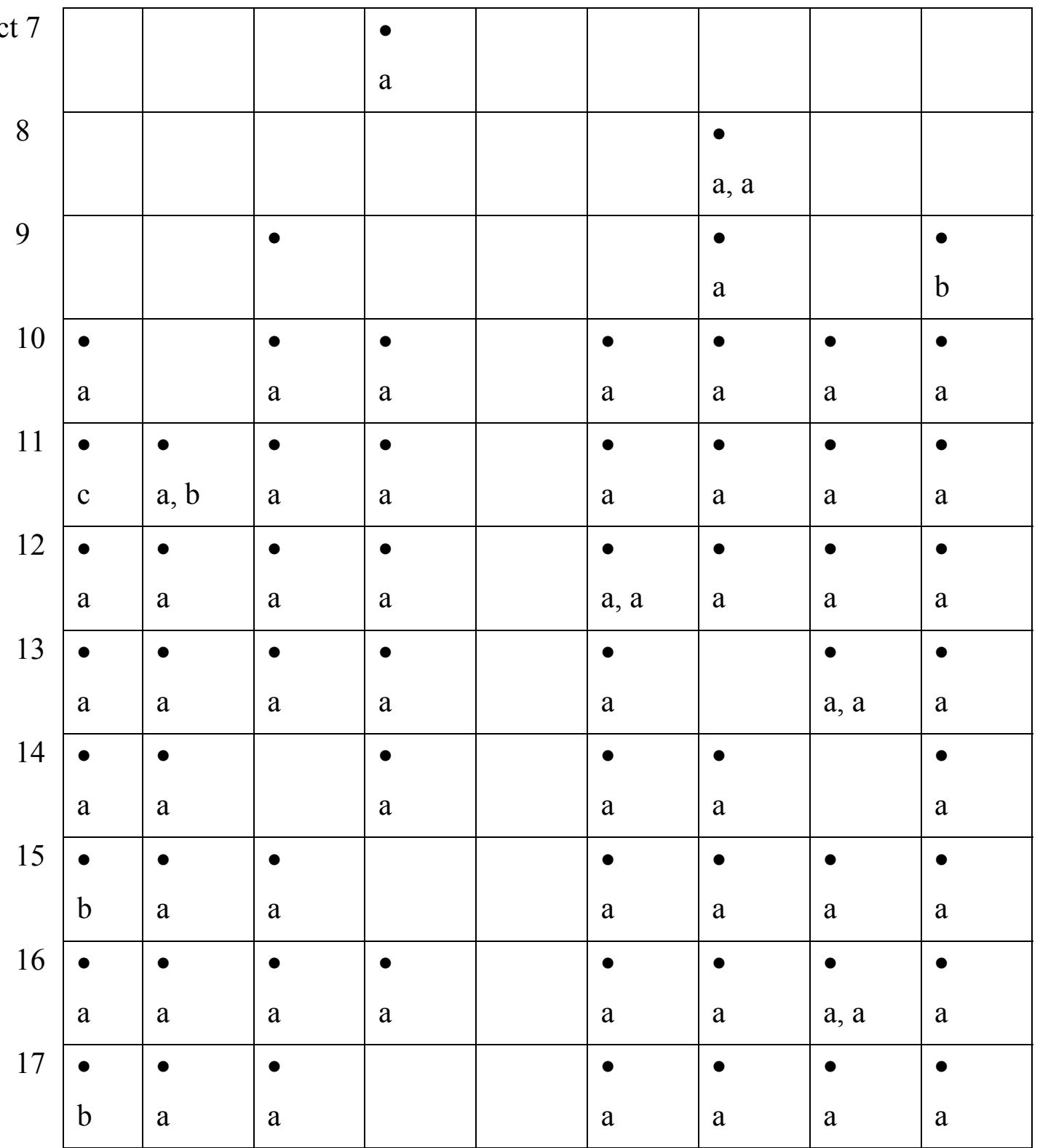

A dot indicates that part of Area 10 is contained in a cluster that is significant at the level indicated based on simulations of clusters in the frontal pole.

Legend: $\mathrm{p}<.0005 ; \mathrm{b} \quad \mathrm{p}<.005 ; \mathrm{c} \quad \mathrm{p}<.05 ; \mathrm{d} \quad \mathrm{p}<.10$

Multiple letters refer to separate clusters of voxels
Component 2

34



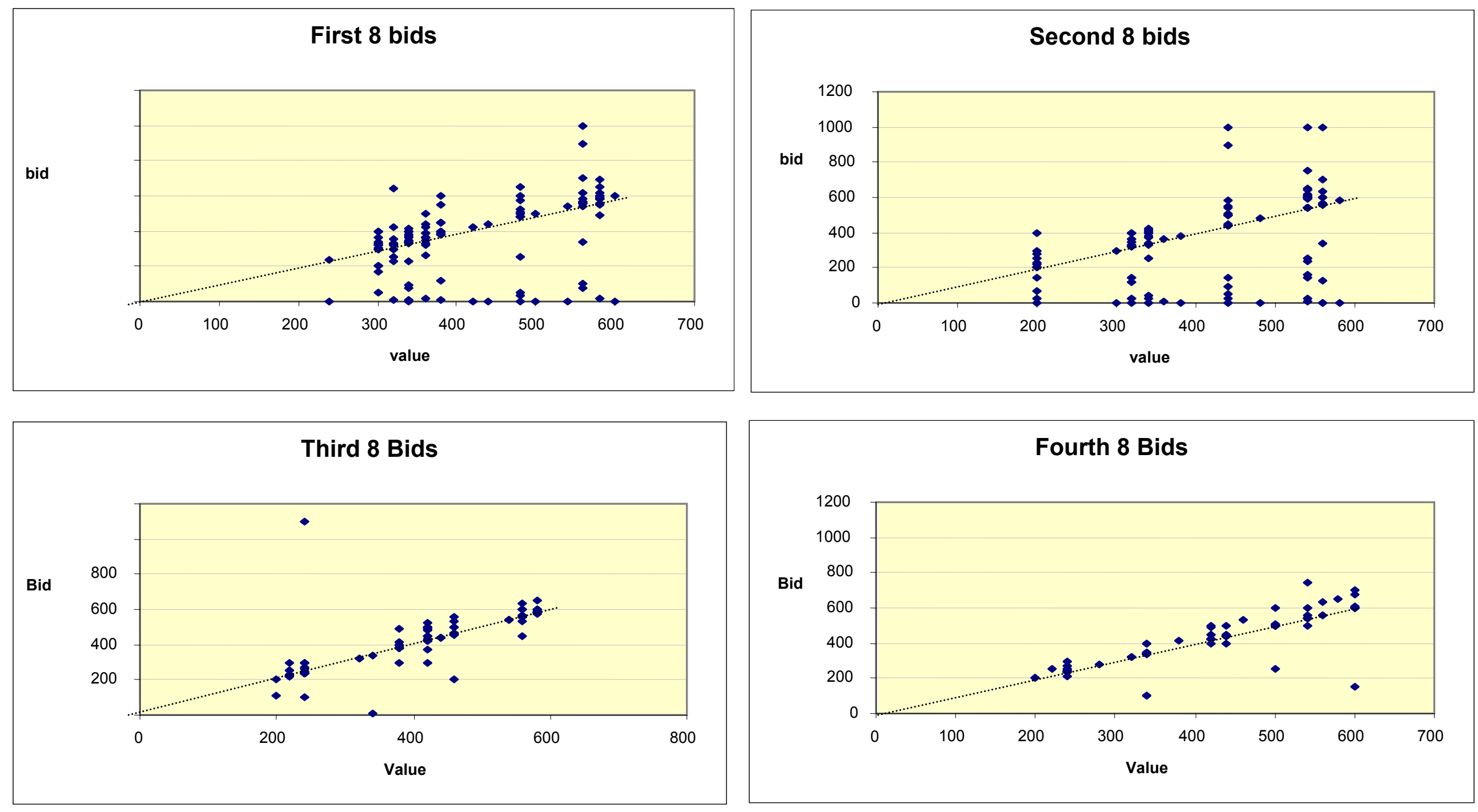

Figure 2. Pooled Bids of all Individuals 
Figure 3
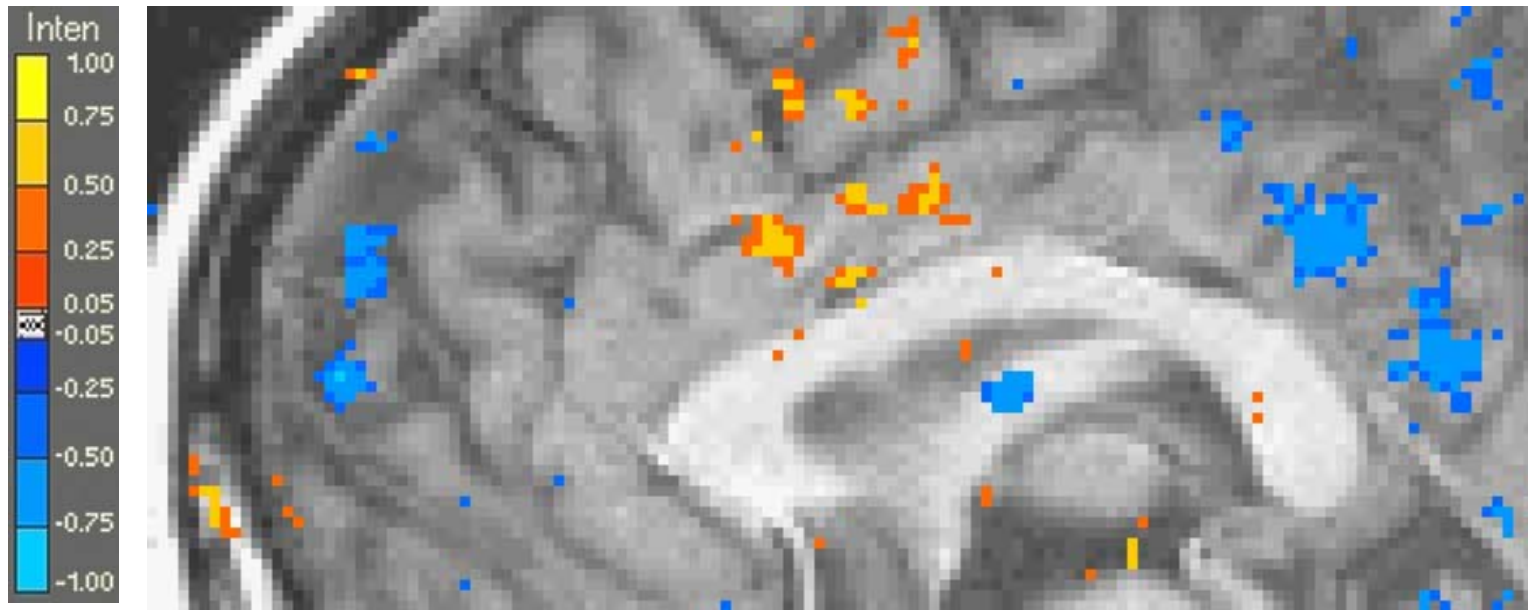

Average response for the first 16 rounds minus the average for rounds 17-32. Note the activity in the anterior cingulate cortex is greater in the first 16 rounds. Sagittal view at $\mathrm{X}=0$.

Figure 4

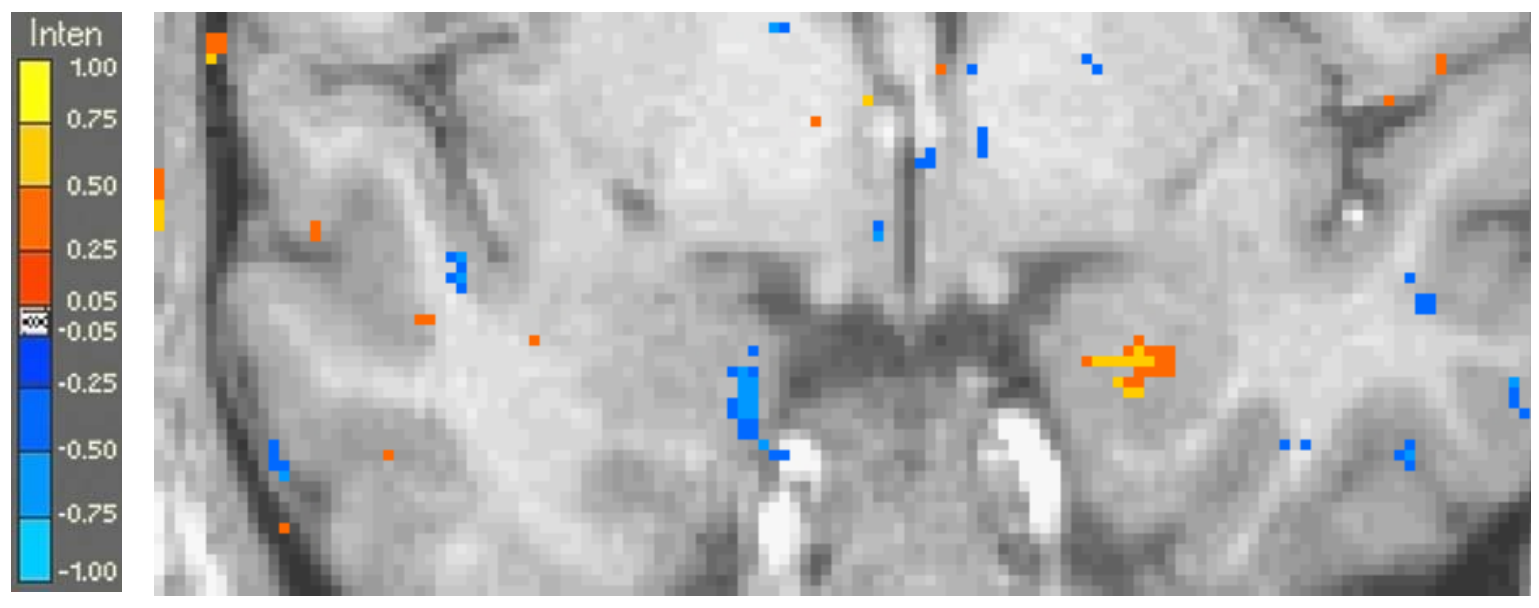

Average response for the first 16 rounds minus the average for rounds 17-32. Note the activity is greater on the right amygdala in for the first 16 rounds, and shifts to the left side for rounds 17-32. Coronal view at $\mathrm{y}=1$. 


\section{Instructions}

This is an experiment in decision-making. During the experiment you will be asked to do several repetitions of a selling task. The experiment will consist of a series of rounds. In each round, you will perform the task once. The amount of money you earn will depend upon the decisions you make.

Our purpose is to study various technical issues involved in decision-making and motor tasks. Your purpose should be to simply make as much money as you can.

\section{Selling Task}

The selling task works as follows. You will receive a coupon worth money to you. You may keep the coupon and collect the money or you can sell the coupon to the experimenter. Your task is to choose a selling price for the coupon and enter it with the keypad.

Your selling price is compared to an offer price randomly generated by the computer. The price chosen by the computer is completely unrelated to your coupon value or your price.

The sale is best described as an auction but it is much different from any auction you have seen before.

If the computer's randomly generated offer price is higher than (or the same as) your selling price, then you sell the coupon at the computer's offer price. For example, if your selling price is 900 , and the computer's offer price is 1000 , then the experimenter buys from you at a price of 1000 .

If the computer's offer price is below your selling price, then the experimenter does not buy from you. You would keep the coupon and collect its value. 
If the computer's random offer price is more than your value of the coupon you would prefer to sell the coupon.

If the computer's random offer price is less than your value of the coupon you would prefer to keep the coupon. 
Money and Sequence

After you have completed each task the screen will display your earnings from the particular task and your accumulated total earnings. You will be paid of the accumulated total earnings. (For technical reasons the numbers used in the experiment are a multiple of the actual money.) After earnings are displayed the screen will go blank for a while and then the next task will be shown.

In order to assure you that the computer's offer prices are completely unrelated to your offer price or your value of the item, the computer's offers have been previously generated. These offer prices are listed in the envelope you have been given. If you want, after the experiment you will be able to verify this independence by comparing the list to the offer prices displayed during the experiment. 
$*$

Points to remember:

1. You enter the lowest price that you are willing to offer the coupon for.

2. If your offer is the same or higher than the computer's offer, you keep the coupon. If your offer is lower, then you sell it at the higher price.

\section{If the computer's offer is higher than}

the coupon value, you make more money selling.

If it is lower, you are better off keeping the coupon.

$*$

Summary of instructions show to subjects after they were positioned in the scanner

The way to make the most money in the experiment is to set your offer price equal to the value of the coupon.

If you do this, then you will never sell the coupon for less than what it will pay at the end of the experiment.

Also, you will never reject a chance to sell the coupon for more than it will pay.

The program will restart now. Your earnings will be added to what you have earned so far. 
Message shown to subjects after they had completed the first sixteen auctions. 